\title{
Linx
}

Revue des linguistes de l'université Paris X Nanterre

$57 \mid 2007$

Études de syntaxe : français parlé, français hors de

France, créoles

\section{« Moi je comprends pas et ça ça m'intrigue »}

Ce que peut nous apprendre la double occurrence de ça dans les énoncés oraux présentant un phénomène de dislocation

\section{Emmanuelle Guerin}

\section{(2) OpenEdition}

\section{Journals}

Édition électronique

URL : http://journals.openedition.org/linx/64

DOI : $10.4000 /$ linx.64

ISSN : 2118-9692

Éditeur

Presses universitaires de Paris Nanterre

Édition imprimée

Date de publication : 1 décembre 2007

Pagination : 27-36

ISSN : 0246-8743

Référence électronique

Emmanuelle Guerin, « " Moi je comprends pas et ça ça m'intrigue » », Linx [En ligne], 57 | 2007, mis en ligne le 15 février 2011, consulté le 15 mai 2020. URL : http://journals.openedition.org/linx/64 ; DOI : https://doi.org/10.4000/linx.64 


\title{
«Moi je comprends pas et ça ça m'intrigue » - Ce que peut nous apprendre la double occurrence de ça dans les énoncés oraux présentant un phénomène de dislocation
}

\author{
Emmanuelle Guerin \\ Université d'Orléans, IUFM Centre Val de Loire \\ - Laboratoire Ligérien de Linguistique et MoDyCo
}

(0) Moi je comprends pas et ça ça m’intrigue

Comme l'illustre l'énoncé (0), ça est un mot qui, dans une construction présentant ce que l'on appelle communément un phénomène de dislocation, peut occuper les deux positions (désormais $\mathrm{P} 1$ et P2), a priori concurrentes, dévolues à deux éléments qui semblent évoquer le sujet d'un même verbe. Dans cet article nous tenterons de montrer en quoi cette spécificité éclaire certains aspects du sens de ça.

A partir de ce que l'on sait des fonctions pragmatiques et sémantiques attribuées aux éléments disloqués, nous tenterons de comprendre pourquoi l'actualisation d'un énoncé tel que (0) semble davantage pertinente dans certaines situations de communication en dehors de toute considération reposant sur l'opposition oral/écrit qui met en avant un prétendu bon usage absolu qui s'illustre essentiellement à l'écrit. Nous reviendrons, par extension, sur la supposée équivalence entre ça et cela : en quoi le fonctionnement de ça est-il indépendant de celui de cela? Partant, l'observation 
d'énoncés tels que (0) constitue un point de départ pertinent puisque manifestement ça et cela n'y sont pas interchangeables.

En somme, nous chercherons à comprendre en quoi l'efficacité ('interprétabilité en situation) d'un tel énoncé, vue sa constitution, est relative à la combi-naison de certains paramètres situationnels : qu'est-ce qui, dans son fonctionnement, permet à ça d'apparaître préférentiellement dans certaines situations ?

\section{Le phénomène de dislocation ${ }^{1}$}

Les constructions disloquées ont la particularité de se caractériser ainsi : «par la coexistence d'une construction verbale complète et autonome, d'un pronom clitique régi par le verbe et d'un élément nominal, pronominal, adjectival ou autre, dit disloqué avant ou après la construction verbale parce qu'il n'est pas possible a priori de lui attribuer une fonction syntaxique traditionnelle. » (Blasco-Dulbecco, 1999, p. 9). Cette description du phénomène amène deux types d'approches : (i) certains envisagent la dislocation comme une forme de pléonasme, de redondance. Dans cette perspective, les deux éléments seraient strictement coréférentiels. Autrement dit, il n'y aurait aucun intérêt communicationnel à évoquer deux fois le même élément. Les grammaires les plus normatives bannissent ce type de constructions. Cette condamnation est largement nourrie du fait que les occurrences de constructions disloquées sont bien plus nombreuses dans les productions orales ou écrites informelles et on sait quel sort est traditionnellement réservé à ce type de productions. Ainsi, on considère que l'élément en P2 n'a aucune fonction expressive : il est envisagé comme soudé au verbe à l'image du clitique ${ }^{2}$. Même si l'on observe que l'élément en P2 est ou fonctionne comme un clitique, on ne peut pas pour autant considérer qu'il n'a pas de rôle à jouer dans le contenu informationnel de l'énoncé. C'est ce qu'on peut voir dans les énoncés suivants :

(1a) Marie quittera jamais Jean-Marc ${ }^{3}$

(1b) Marie elle quittera jamais Jean-Marc

L'interprétation des deux énoncés est sensiblement différente : dans les deux cas, Marie renvoie à la personne de sexe féminin que l'on appelle « Marie »; en (1b), elle renvoie à ce que l'on sait de la personnalité de Marie (ce qui fait qu'elle ne quittera

\footnotetext{
${ }^{1}$ Il n'est pas question dans cet article de proposer une étude fine du phénomène de dislocation dans toutes ses réalisations. Nous limitons nos propos aux constructions telles que celle repérable en (0) étant donné la finalité de cette étude. C'est donc à partir de ce qui peut être dit de la dislocation en général que nous chercherons à décrire ce qui se passe en (0).

${ }^{2}$ Heap et Roberge (2001, p. 64) définissent les clitiques comme : «des éléments qui fonctionnent comme des arguments d'un prédicat, mais qui se comportent plus comme des morphèmes dépendants que comme des lexèmes ».

${ }^{3}$ Enoncés recueillis à la volée.
} 
jamais Jean-Marc) ${ }^{4}$. La distinction que l'on peut faire de ces deux énoncés, aussi fine soit-elle, montre que la présence d'un clitique en P2 n'est pas exclusivement systématique. Si tel était le cas, les constructions sans éléments détachés tendraient à disparaitre. Or, comme le montre Lambrecht (1987), les constructions telles que (1a) n'ont aucune fonction pragmatique à l'oral. Autrement dit, (1a) est interprétable sans que se mettent en place un jeu d'inférences, une collaboration active des acteurs de l'échange, sans que l'on ait à activer le référent visé par «Marie » à partir d'informations autres que celles données en cotexte. Cette absence de fonction pragmatique est en soi une fonction et on comprend alors que produire (1a) ou produire (1b) n'est pas le fruit du hasard mais est motivé par un projet intentionnel précis s'appuyant notamment sur l'appréhension du savoir partagé avec l'autre: pour produire 1(b), il faut s'assurer que l'autre connaît suffisamment Marie. Traiter le phénomène de dislocation en termes de redondance, conduit à ne pas tenir compte de la portée communicative (intention du locuteur/scripteur et interprétabilité en situation de l'énoncé) d'une construction disloquée.

(ii) En adoptant un autre point de vue, les deux éléments occupant les deux positions (P1 et P2) peuvent être perçus comme ayant chacun une véritable fonction distincte. Cette seconde approche pose l'élément disloqué comme introducteur du thème de l'énoncé. "J'appelle phrase segmentée un énoncé divisé en deux parties séparées par une pause et dont l'un, désigné ici par Z, est le but de l'énoncé, le prédicat psychologique, le propos, et l'autre, figuré par $A$, le sujet psychologique, le thème qui sert de base au propos. (...) L'ordre des termes est $A Z$ ou $Z A$ : comparez cette lettre (A), je ne l'ai pas reçue $(Z)$ et je ne l'ai jamais reçue (Z), cette lettre (A). » (Bally, 1941, p. 36). La fonction de l'élément disloqué est donc en lien avec la fonction du thème dans un énoncé. L'information donnée a les propriétés que l'on attribue généralement au thème. Observons le tableau suivant inspiré du travail de Blasco-Dulbecco dans lequel on liste l'ensemble des propriétés, qualités, attributs du thème et du propos que la littérature sur le sujet propose :

\begin{tabular}{|c|c|}
\hline Thème & Propos \\
\hline Ce dont on parle & Ce qu'on en dit \\
\hline Ancien & Nouveau \\
\hline Donné & Non-donné \\
\hline Connu & Non-connu \\
\hline Présupposé & Focalisé \\
\hline & Emphatique \\
\hline Point de départ & But \\
\hline Support & Apport \\
\hline Fond & Figure \\
\hline Récupérable & Non-récupérable \\
\hline Prévisible & Non-prévisible \\
\hline Activé & \\
\hline Saillant & \\
\hline
\end{tabular}

\footnotetext{
4 1(b) équivaut à : Marie, étant donné ce que nous savons d'elle, ne quittera jamais Jean-Marc. En 1(a), le locuteur affirme que Marie ne quittera jamais Jean-Marc sans se préoccuper de ce que sait l'interlocuteur des arguments en faveur de ses propos : il détient la vérité.
} 


\begin{tabular}{|c|c|}
\hline Centre d'intérêt & \\
\hline Lié à la conscience immédiate & \\
\hline Lié au contexte & Indépendant du contexte \\
\hline Liés aux circonstances & \\
\hline Notoire & \\
\hline
\end{tabular}

Comme il apparait dans ce tableau, un thème est donc une information donnée, récupérable, activée, liée à la conscience immédiate, au contexte, aux circonstances. Nous insistons particulièrement sur ces critères car, comme nous le verrons, ils seront déterminants quant aux hypothèses que nous poserons par la suite. Ainsi, il nous importe de nous intéresser à la relation entre l'élément en P1 et les informations connues (par exemple, les informations repérables dans le texte en amont (de Fornel, 1988)) avant de nous intéresser à la relation entre l'élément en P1 et l'élément en P2 ${ }^{5}$. Proposer un énoncé présentant un phénomène de dislocation c'est permettre au récepteur de réactiver des informations repérables en dehors de cet énoncé mais indispensables pour la bonne interprétation de celui-ci. On comprend ainsi le nombre d'énoncés présentant un phénomène de dislocation à l'oral : cette relation permet au locuteur de réactiver la saillance d'un élément évoquée par ailleurs (implicite ou préalablement suggérée) qui pourrait être perdue par l'interlocuteur du fait de l'absence de trace laissée à l'oral (en situation de lecture, les retours en arrière permettent au lecteur le repérage des éléments saillants). Comme le souligne Bally (cité par Galazzi, 1994, p. 35-36) : "La dislocation est un stimulant pour l'attention, que l'ordre logique endort, au contraire, sur un oreiller de paresse.». Partant, considérer le phénomène de dislocation comme une simple redondance n'est pas suffisant étant donné la fonction pragmatique que l'on attribue à l'élément disloqué.

\section{2. ça : une autonomie référentielle relative}

Si ça peut apparaître en position disloquée, alors on ne peut pas assimiler son fonctionnement à celui d'un clitique, c'est-à-dire un simple morphème verbal. Ça est également à dissocier des éléments de type moi, toi, lui puisque contrairement à eux, il a la possibilité de constituer un sujet grammatical.

(2a) Ça m'aide beaucoup.

(2b) *Toi m'aides beaucoup.

Son comportement est davantage à rapprocher de celui des SN. En effet, un SN peut apparaître en position disloquée et, dans des constructions simples, être sujet grammatical.
(3a) Paul m'aide beaucoup
(3b) Paul il m'aide beaucoup

\footnotetext{
${ }^{5}$ En centrant l'analyse sur cette relation, en excluant celle qui unit l'élément en P1 et les informations partagées, on aboutit nécessairement au constat de redondance.
} 
Une différence cependant, les SN ne peuvent être le sujet strictement grammatical dans les énoncés présentant un phénomène de dislocation, autrement dit être perçus comme des morphèmes. S'ils entrent en combinaison avec un autre élément comme c'est le cas de l'élément disloqué et du sujet grammatical, ils ne peuvent prendre la position de l'élément dont la charge sémantique serait moindre.

(4) *Lui, Paul m'aide beaucoup

Alors comment expliquer cette différence ? Qu'est-ce qui oppose un SN à ça?

Un SN est une unité référentiellement autonome. Moeschler (1994, p. 134) précise: «le terme autonomie référentielle désignant justement la possession de la signification lexicale et la capacité d'une expression qui en est pourvue à déterminer, par elle-même, son référent. ». L'autonomie référentielle des SN permet l'introduction du thème en évoquant une réalité du monde unique. Il ne peut donc être le relais d'une unité dont la charge sémantique est nécessairement moins importante. De fait, ça serait suffisamment autonome référentiellement pour apparaître en position disloquée, mais susceptible de servir de relais et se combiner au verbe afin d'assurer la complétude syntaxique et sémantique d'une construction prédicative. En d'autres termes, ça peut être interprété sans que l'on ait recours au repérage d'un antécédent en cotexte au même titre qu'un SN. C'est ce qui permet à l'un et l'autre d'introduire le thème d'un énoncé en position disloquée. En revanche, son autonomie référentielle n'est pas totale puisqu'il ne renvoie pas à une réalité unique dans le monde. Cette relative autonomie référentielle pourrait démentir qu'il y ait redondance de la double occurrence de $c ̧ a$. On peut donc poser l'hypothèse suivante : ça (P1) pose le thème et ça (P2) sert de relais à ce thème dans la construction prédicative. Il ne s'agit pas de considérer qu'il y aurait deux ça. Le sens de ça serait tel que sa position dans l'énoncé serait un facteur de relativisation de son autonomie référentielle.

\section{3. ça : interprétation et contexte}

Du fait de sa relative autonomie référentielle par opposition à l'autonomie référentielle absolue des $\mathrm{SN}$, l'interprétation de ça est dépendante, mais dépendante de quoi ? L'environnement discursif ne fournit pas d'éléments suffisants à l'interprétation de ça. Si l'on observe l'énoncé suivant:

\section{(5) Et Paul, ça va?}

Il ne s'agit pas d'interroger la capacité de Paul à «aller» mais plutôt de considérer par exemple la santé ou le moral de Paul. On évoque là une dépendance à l'autre source d'informations à la disposition des acteurs d'un échange pour interpréter les unités d'un énoncé. En l'occurrence, le contexte fournit un certain nombre d'informations nécessaires à l'interprétation d'un énoncé. Par « contexte», on entend ce qui est perceptible ou implicitement connu par les interactants au moment de l'échange. Un tel énoncé peut être produit à condition que la santé ou le moral de Paul soit une préoccupation actuelle, partagée et saillante (Guerin, 2006).

Il y a donc les informations fournies par le cotexte et celles fournies par le contexte. Si ça est référentiellement autonome vis à vis des éléments du cotexte, nous 
postulons que son interprétation est dépendante du contexte. En tant qu'élément référentiellement dépendant, ça peut renvoyer à des réalités différentes et ainsi se comporter en simple relais. En tant qu'élément référentiellement indépendant du cotexte, ça peut renvoyer par lui même à une réalité unique dans le monde tel qu'il est perçu par les interactants et ainsi apparaître en position disloquée.

Notre hypothèse se renforce si l'on s'appuie sur le fait que ça est davantage utilisé, et surtout utilisé de façon pertinente, dans les productions issues de situations de communication de type face à face, c'est-à-dire celles permettant aux interactants d'entretenir une forte connivence. En effet, pour qu'un ça soit efficacement interprété, il est nécessaire que les deux partenaires aient une appréhension commune du contexte. $C a$ en $\mathrm{P} 1$ n'est possible qu'à la condition qu'il renvoie à un référent suffisamment stable (à l'image du $\mathrm{SN}$ ) pour les deux partenaires. On ne saurait s'assurer de cette stabilité sans un partage évident d'un certain nombre d'informations. Considérons l'énoncé suivant :

(6) les filles ça joue pas au foot

(6) est pertinent à la condition que l'on partage l'idée que «les filles» ne sont pas faites pour le sport, ne comprennent rien au foot, n'ont pas les capacités pour jouer au foot, etc. Sans ce partage, «les filles » renvoient à des êtres humains de sexe féminin et en tant que tels, rien ne justifie qu'ils ne «jouent pas au foot» puisqu'ils sont dotés de jambes, de pieds et d'un cerveau, éléments requis pour la pratique du foot-ball. En somme, le sens de $c ̧ a$, son mode de référencement, implique que la situation de communication permette le partage d'informations nécessaires à l'activation du référent tel qu'envisagé au moment de l'élaboration d'un énoncé. On comprend dès lors que si ça apparait plus souvent en situation de communication orale c'est parce que ces situations favorisent la connivence des interactants et non parce qu'il s'agirait d'une unité relevant strictement de l'oral comme on peut le lire ici ou là. En posant que ça relèverait de l'oral on impose d'emblée l'équivalence avec une unité qui relèverait de l'écrit standard. Étant donné « l'idéologie du standard» telle qu'elle se manifeste dans nos sociétés, cette équivalence s'accompagne d'un jugement de valeur qui tend à invalider ça au profit de cela. Or, les conditions requises pour un usage pertinent de $c a$ ne sont pas les mêmes que pour cela. Ainsi, en entretenant l'idée qu'il y aurait de telles équivalences, on perd de vue la dimension pragmatique.

\section{4. ça et cela}

De fait, la supposée équivalence de ça et cela est à reconsidérer, une fois qu'on a écarté l'idée que l'opposition entre les deux unités repose sur le caractère oral de l'un et écrit de l'autre. En revanche il est possible d'éclairer les éléments qui motivent leur distribution en considérant les implications pragmatiques de l'emploi de chacun. Pour autant, toutes les occurrences de ça et cela ne sont pas analysables en ces termes : en usage, la sélection de l'un ou l'autre est souvent motivée par la valeur symbolique qui leur est attribuée. Cela est envisagé comme la forme correcte en toute circonstance, même lorsque ça semblerait plus approprié. C'est la survalorisation de la forme standard et des unités qui la composent qui entraîne cette tendance. On peut alors 
parler d'un phénomène d'hypercorrection fréquent, notamment au moment de la scolarisation, c'est-à-dire au moment où l'on entre de plein pied dans l'idéologie du standard. Comme le souligne Berrendonner (1988: 60-61) : « [les apprenants] viennent à user des formes an seul vu de leur valeur de prestige, en faisant fi de toutes les autres particularités. ». Ainsi, si l'analyse que nous présentons ici tend à éclairer le sens de $̧ a$, et par extension celui de cela, nous ne perdons pas de vue qu'en usage, le choix de ça ou cela se fait bien souvent en dépit de ce sens. Cette considération d'ordre sociolinguistique est nécessaire pour expliquer certaines occurrences de ça ou cela dont l'analyse semblerait invalider notre hypothèse.

Plutôt que d'affirmer que cela est l'unité correcte, prestigieuse, parce qu'elle illustre la forme standard, il est possible d'en proposer une description qui prenne en charge la concurrence avec $c ̧ a$ sans s'appuyer sur les jeux de valeurs symboliques. En l'occurrence, la non autonomie référentielle de cela vis à vis du cotexte et du contexte ${ }^{6}$ impose que son emploi soit toujours accompagné d'une explicitation du référent visé. Cette démarche d'explicitation des déictiques est propre aux productions issues de situations de communication ne permettant qu'une connivence réduite entre acteurs, c'est-à-dire les situations de communication dont les éléments constitutifs ne permettent pas le partage de beaucoup de connaissances. Nous proposons de parler de « mémoire discursive » ${ }^{7}$ faible, ou, dans les termes de Koch et Esterreicher (2001), de situations relevant de la distance communicative. C'est le cas de la plupart des productions écrites. Ce n'est pas pour autant que l'on doit en conclure un partage strict entre ce qui relève de l'oral et ce qui relève de l'écrit. Si complémentarité il y a entre ça et cela, elle réside dans le fait que lorsque la connivence est forte on préfèrera ça qui, par sa dépendance au contexte appréhendé communément, renforce la coopération ${ }^{8}$.

Comment se manifeste cette différence ? Pour mettre en lumière ce qui se joue référentiellement pour ça et cela, partons de deux expressions courantes qui ne semblent pas supporter la substitution : cela dit et ça marche dans les énoncés (7), extrait des propos d'un manifestant interrogé par un journaliste de France Info, entendu à la radio et (8), récolté à la volée.

(7a) le gouvernement a l'air de vouloir faire des efforts mais cela dit c'est toujours les mêmes qui trinquent.

(7b) *le gouvernement a l'air de vouloir faire des efforts mais ça dit c'est toujours les mêmes qui trinquent.

(8a) tu branches le câble USB et après tu suis les indications et ça marche

\footnotetext{
${ }^{6}$ Cela a un fonctionnement typique des pronoms (autres qu'indéfinis): son interprétation passe nécessairement par la récupération d'un référent appréhendable en co- ou contexte sans que ce dernier subisse la moindre modification lors de son activation par cela.

${ }^{7}$ La mémoire discursive se constitue de l'ensemble des connaissances valides au moment de l'échange et partagées par les acteurs de la communication (Berrendonner, 1983).

${ }^{8} \mathrm{Par}$ ailleurs, on comprend que le caractère prestigieux qui peut être attribué à cela, au dépens de ça, tient à la nature des situations de communication dans lesquelles il est pertinent de l'actualiser : la plupart des situations relevant de la distance communicative sont des situations caractérisées par un fort enjeu social.
} 
(8b)* tu branches le câble USB et après tu suis les indications et cela marche ${ }^{9}$

En (7a), cela permet l'activation de «le gouvernement a l'air de vouloir faire des efforts». Cela, dans l'expression cela dit, permet de réintroduire une information telle qu'elle a été donnée sans suggérer une quelconque modification.

Quant à « ça marche», nous renvoyons à ce que nous disions de l'énoncé (5): ce qui «marche» n'est pas «tu branches le câble USB» mais bien tout le branchement tel qu'il est perceptible par les deux partenaires.

On pourrait dire qu'il s'agit dans les deux cas de locutions figées. Si c'est un argument valable, on peut cependant s'interroger sur les raisons d'une telle résistance alors que la plupart des grammaires voient en $c ̧$ la forme actuelle de cela ${ }^{10}$. Notre hypothèse conduit à considérer que les deux locutions ne permettent pas la substitution étant donné le sens de ça. Comme le souligne Cadiot (1987, p. 174) : «ça ne peut être considéré comme une forme de reprise (anaphore) à l'identique, de cet antécédent supposé, mais bien plus comme la trace d'un accès propositionnel à sa référence (à ce dont l'antécédent "nous parle » dans un énoncé donné). Même lorsqu'il y a bien reprise, l'essentiel est que cette reprise (considérée ici comme un processus) ne traite pas son antééédent comme une «valeur», mais comme l'argument d'une ou plusieurs proposition(s) implicite(s), plus ou moins accessibles et variablement explicitables. ». En somme, ça ne permet pas l'activation de l'information telle qu'elle est ou telle qu'elle est dite : l'information est présentée à partir de ce que le locuteur/scripteur cherche à en dire («trace d'un accès propositionnel»). En (8a), «le câble USB» est le point d'appui de l'interprétation de ça mais il ne constitue pas pour autant toute la référence. Les éléments qui la complètent sont à chercher dans les connaissances partagées par les interlocutrices au moment de l'échange (la perception visuelle de l'installation et la connaissance de l'objectif de raccorder les deux machines).

Dès lors, on peut regarder autrement les occurrences de ça et cela dans des constructions qui supportent la substitution, comme dans ces énoncés forgés :

(9a) la mairie installe des vélos en libre accès. Cela ne durera pas longtemps.

(9b) la mairie installe des vélos en libre accès. Ça ne durera pas longtemps.

En (9a), cela réfère strictement à la première phrase et on comprend que ce qui ne durera pas c'est l'installation des vélos. En revanche, en (9b), ça permet une autre interprétation : c'est l'activité autour des vélos en libre accès qui ne durera pas (« c'est une mode passagère »). Pour que cette seconde interprétation soit possible, il faut que le récepteur ait connaissance de ce en quoi consiste l'activité. Sinon, c'est la première interprétation qui s'impose, celle que permet cela.

\footnotetext{
${ }^{9}$ Cet énoncé est invalide avec le sens de (8a). (8b) pourrait s'entendre avec un sens différent: ce serait alors l'ordinateur pointé du doigt par le locuteur qui marche et non l'ensemble du système.

10 On observe effectivement une diminution des emplois de cela au profit de ça dans les pratiques langagières contemporaines. Cependant, il se peut que cet état de fait résulte d'une modification des pratiques (notamment en lien avec les avancées technologiques) qui favorise les situations de communication renforçant la connivence et la coopération des locuteurs/scripteurs. La distance communicative tend à se raréfier, et les faits de langue pertinents dans les situations de proximité situationnelle tendent à l'emporter.
} 
Ce dernier exemple permet de revenir sur la question à l'origine de ce texte, la double occurrence de ça dans les constructions disloquées. ça a un sens qui lui permet selon le contexte de fonctionner :

- $\quad$ comme un anaphorique, en P2, lorsque les seuls éléments qui permettent la restitution du référent sont à chercher en cotexte (seules informations alors partagées par les acteurs de la communication) ${ }^{11}$;

- comme une unité permettant l'activation d'un référent tel qu'il est considéré par le locuteur au moment de la production d'un énoncé, en P1. Pour que ça soit bien interprété, il faut donc que le récepteur ait connaissance des éléments concernés ${ }^{12}$.

Autrement dit, on retrouve les deux niveaux d'autonomie référentielle qui permettent à ça d'occuper les deux positions caractéristiques des constructions disloquées.

L'étude du phénomène de dislocation et la particularité de P1 et P2 permet de révéler certaines propriétés des unités susceptibles d'occuper ces positions qui ne seraient pas forcément observables en dehors de ce contexte syntaxique. Ainsi, le comportement de ça, la double occurrence de ça, dans les énoncés présentant un phénomène de dislocation met en lumière une caractéristique de son comportement référentiel : le lien manifeste avec le contexte.

Nous nous inscrivons ici dans la question plus large du rapport entre description de la langue et considération de l'oral et de l'écrit. On voit à partir de cet exemple que la description des faits de langue observables principalement dans les énoncés provenant de situations de communication autres qu'appelant l'actualisation de la forme standard écrite du français, est souvent lacunaire voire absente. Si on se place dans une perspective qui n'est pas contrainte par la dichotomie écrit/oral, correct/incorrect, bon/mauvais usage, on peut proposer une description grammaticale davantage en lien avec les usages réels. Il est de fait possible d'intégrer le niveau pragmatique à l'analyse, niveau souvent exclu de la description grammaticale traditionnelle : l'opposition oral/écrit sert de caution pour la mise à l'écart de faits de langue qui contredisent les règles élaborées à partir de productions relevant de la forme standard.

\footnotetext{
${ }^{11}$ Ce qui explique la possible équivalence de ça et cela dans les énoncés (9).

12 En (9b) la connaissance partagée concerne l'usage des vélos en libre service et ce que sait le locuteur de l'activité qui s'organise autour.
} 


\section{RÉFÉRENCES BIBLIOGRAPHIQUES}

BALLy, C. (1941), «Intonation et syntaxe », Cabiers F. de Saussure, I, pp. 33-42.

Berrendonner, A. (1983), «Connecteurs pragmatiques et anaphores », Cabiers de Linguistique française, $\mathrm{n}^{\circ} 5$, pp. $215-246$.

Berrendonner, A., (1988), "Normes et variations » in Schoeni, G. et al. (dir.) La langue française est-elle gouvernable?, Neuchâtel-Paris, Delachaux et Niestlé.

Blasco-Dulbecco, M. (1999), Les dislocations en français contemporain. Etude syntaxique, Paris, Champion.

CADiot, P. (1987), «De quoi ça parle ? A propos de la référence de ça, pronom-sujet », Le français moderne, $\mathrm{n}^{\circ} 56$, pp. 174-192.

ForNEL, M. (de) (1988), «Constructions disloquées, mouvement thématique et organisation préférentielle dans la conversation », Langue française, $\mathrm{n}^{\circ} 78$, pp. 101-123.

GALAZZI, E. (1994), «L'ordre des mots en français » in La grammatica francese e il suo insegnamento, Brescia, Quaderni del CLUC.

GUERIN, E. (2006), Introduction de la notion de variation situatiolectale dans la grammaire scolaire par la caractérisation de deux opérateurs pragmatiques: on et ça, Thèse de doctorat sous la dir. de F. Gadet, soutenue à l'université Paris X.

HeAp, D. \& Roberge, Y. (2001), « Cliticisation et théorie syntaxique. 1971-2001 », Revue québécoise de linguistique, $\mathrm{n}^{\circ}$ 30, 1, pp. 63-90.

KOCH, P. \& OESTERREICHER, W. (2001), «Langage parlé et langage écrit », Lexikon der romanistischen Linguistik, tome 1, pp. 584-627, Tübingen, Max Niemeyer Verlag.

LAmbrecht, K. (1987), "On the status on S-V-O sentences in French discourse » in Tomlin, R.S. (éd.) Coberence and Grounding in Discourse, Amsterdam, J. Benjamins.

Moeschler, J. \& Reboul, A. (1994), Dictionnaire Encyclopédique de Pragmatique, Paris, Le Seuil. 